\title{
Adiponectin circulating levels and 10-year (2002-2012) cardiovascular disease incidence: the ATTICA Study
}

Ioannis Kyrou ${ }^{1,2,3,4}$, Olga Tsantarlioti ${ }^{1}$, Demosthenes B. Panagiotakos ${ }^{1}$, Constantine Tsigos ${ }^{1}$, Ekavi Georgousopoulou ${ }^{1}$, Christina Chrysohoou ${ }^{5}$, Ioannis Skoumas ${ }^{5}$, Dimitrios Tousoulis ${ }^{5}$, Christodoulos Stefanadis ${ }^{5}$, Christos Pitsavos ${ }^{5}$; and the ATTICA study group

${ }^{1}$ Department of Science of Dietetics and Nutrition, School of Health Science and Education, Harokopio University, Athens, Greece; ${ }^{2}$ Aston Medical Research Institute, Aston Medical School, Aston University, B4 7ET, Birmingham, UK; ${ }^{3}$ Translational \& Experimental Medicine, Division of Biomedical Sciences, Warwick Medical School, University of Warwick, CV4 7AL, Coventry, UK; ${ }^{4}$ WISDEM, University Hospitals Coventry and Warwickshire NHS Trust, CV2 2DX, Coventry, UK; ${ }^{5}$ First Cardiology Clinic, School of Medicine, University of Athens, Athens, Greece

\section{Corresponding author}

Professor Demosthenes B. Panagiotakos

Harokopio University in Athens

17671 - Athens, Greece

Tel: +30 210-9549332

E-mail: dbpanag@hua.gr 


\section{Abstract}

Purpose: Adiponectin is an adipokine with anti-inflammatory and cardiovascular-protective properties. Existing epidemiological evidence is conflicting on the exact relationship between adiponectin and long-term cardiovascular disease (CVD) risk. Our aim was to prospectively assess whether circulating adiponectin is associated with long-term incident CVD. Methods: A population-based, prospective study in adults ( $>18$ years) without previous CVD history (ATTICA study). Circulating total adiponectin levels were measured at baseline (2001-2002) in a sub-sample ( $n=531$; women/men: 222/309; age: $40 \pm 11$ years) of the ATTICA cohort and complete 10-year follow-up data were available in 366 of these participants (women/men: 154/212; age: $40 \pm 12$ years). Results: After adjusting for multiple factors, including age, sex, body mass index, waist circumference, smoking, physical activity, Mediterranean diet adherence, hypertension, diabetes and hypercholesterolemia, our logistic regression analysis indicates that an increase in circulating total adiponectin levels by 1 unit was associated with 36\% lower CVD risk (relative risk [RR]: 0.64, 95\% confidence interval [CI]: 0.42-0.96; $\mathrm{p}=0.03)$. Further adjusting for interleukin- 6 plasma levels had no significant impact (RR: 0.60, 95\% CI: 0.38-0.94; $\mathrm{p}=0.03$ ), while additional adjustment for circulating C-reactive protein (CRP) modestly attenuated this association (RR: 0.63, 95\% CI: 0.40-0.99; $\mathrm{p}=0.046$ ). Conclusions: In our study elevated circulating total adiponectin levels were associated with lower 10-year CVD risk in adults without previous CVD, independently of other established CVD risk factors. This association appeared to be modestly attenuated by CRP, yet was not mediated by interleukin-6 which is the main endocrine/circulating pro-inflammatory cytokine.

Key words: adiponectin, cardiovascular disease, CVD risk, ATTICA study 


\section{Introduction}

Adipose tissue secretes in the circulation multiple proteins and factors, collectively termed as adipokines [1, 2]. Circulating adipokines exert various effects on target tissues/organs, thus regulating energy homeostasis, metabolism, insulin sensitivity and cardiovascular functions [3, 4]. Adiponectin (initially reported as Acrp30, AdipoQ, apM1 and GBP28) is a secretory protein encoded by the ADIPOQ gene and is synthesized/secreted almost exclusively by adipocytes, constituting one of the most abundant circulating adipokines (approximately $0.01 \%$ of the total plasma protein) [5-7].

Compiling experimental evidence has shown that adiponectin plays a pleiotropic protective role, exhibiting unique anti-inflammatory, insulin-sensitizing, anti-atherogenic and cardiovascular-protective properties [4-7]. Notably, in contrast to most other adipokines, adiponectin secretion decreases with increasing adiposity [2-7]. As such, the chronic, lowgrade, systemic inflammation, which is caused by increased adiposity and is associated with cardiovascular disease (CVD), is characterized not only by increased circulating proinflammatory biomarkers, such as C-reactive protein (CRP) [8], and interleukin-6 (IL-6) [9], but also by lower adiponectin plasma levels [5-7].

Despite the strong experimental evidence supporting the protective cardio-metabolic role of adiponectin, the epidemiological data on the exact relationship between adiponectin and long-term CVD risk are inconsistent and conflicting [10]. Indeed, in 2004, Pischon et al. showed that high plasma adiponectin levels were associated with significantly lower myocardial infarction risk in middle-aged, CVD-free men of the Health Professionals Followup Study over a 6-year follow-up period [11]. However, other prospective studies have failed to identify a strong, independent association between circulating adiponectin and coronary heart disease (CHD) [12]. Moreover, data from studies in elderly participants or patients with 
CHD or heart failure have reported a paradoxical positive association between increasing adiponectin plasma levels and mortality [10, 13-15].

It becomes evident that long-term prospective studies are still needed in this field. Moreover, the available epidemiological data on this association from prospective cohorts in Mediterranean countries are rather limited. With the aforementioned in mind, the aim of the present work was to prospectively assess whether circulating adiponectin levels are associated with long-term CVD risk in Greek adults from the general population without previous CVD.

\section{Materials and Methods}

\section{Study sample}

The ATTICA study is a population-based, prospective survey conducted in the Attica region of Greece. The detailed ATTICA study design has been previously described in the initial methodology paper [16]. Briefly, 3042 free-living adults ( $>18$ years old; women/men: 1528/1514) without previous CVD history were recruited during 2001-2002 through a multistage, random sampling protocol that selected one participant per household. Comprehensive baseline assessments, including a physical examination and measurements of key biochemical parameters, were performed in all study participants. To address the research question of the present work, in a random sub-sample of 531 study participants (women/men: 222/309; age: $40 \pm 11$ years) we additionally measured circulating total adiponectin levels. No significant differences were observed between the sub-sample of the present study and the total study population regarding the distributions of age, gender, physical activity status, smoking and dietary habits (all p-values $>0.30$ ). The ATTICA study protocol was approved by our Institutional Ethics Committee and the study was conducted according to the World 
Medical Association Declaration of Helsinki. Informed consent was obtained from all individual participants included in the study.

\section{Baseline study assessments}

The baseline study data included demographic characteristics (e.g. age, sex and years of education), personal/family history of CVD, hypertension, hypercholesterolemia and diabetes, as well as detailed information on dietary and key lifestyle habits (e.g. smoking and physical activity), as previously described [16]. Briefly, the dietary evaluation for all study participants was performed using a validated semi-quantitative food-frequency questionnaire, i.e. the EPIC-Greek questionnaire [17], which was kindly provided by the Unit of Nutrition of Athens Medical School, while adherence to the Mediterranean diet was also assessed through the MedDietScore (range 0-55; higher score values indicate better adherence) [18]. Furthermore, the International Physical Activity Questionnaire (IPAQ) was utilized, as an index of weekly energy expenditure, to assess the physical activity level of study participants (according to the IPAQ participants were classified as inactive, minimally active and healthy active) [19]. Moreover, in the context of this study, current smokers were defined as those smoking at least one cigarette per day, while ex-smokers as those who had stopped smoking for at least one year prior to study enrollment. Pack-years of smoking were also calculated as the product of average cigarettes smoked per day by the years of smoking.

Anthropometric parameters were measured for all participants, including height which was measured to the nearest 0.5 centimeter $(\mathrm{cm})$ with a right-angles triangle resting of the participant's scalp and against the wall (participant without shoes and in the upright position with the back square against the measuring wall tape); weight (kilograms; kg) which was measured with a lever balance (participant in light undergarments and without shoes); and waist circumference $(\mathrm{cm})$ which was measured with an inelastic measuring tape at the minimum circumference between the superior iliac crest and the lower margin of the last rib 
(participant in the upright position and at the end of normal expiration). For each participant, the body mass index (BMI) was also calculated as body weight in kilograms divided by the square of the height in meters $\left(\mathrm{kg} / \mathrm{m}^{2}\right)$. Although useful to avoid overestimating BMI in subjects older than 65 years, no particular formulas were adopted for height estimation in study participants over 65 years old. Resting arterial blood pressure (BP; mean of three recordings) in a sitting position was also measured for each participant, and participants with average $\mathrm{BP} \geq 140 / 90 \mathrm{mmHg}$ [or on antihypertensive medication(s)] were classified as hypertensive. Blood samples were collected from each participant at 8-10 am, following 1012 hours of overnight fasting (no food and alcohol intake with the exception of water). Accordingly, serum and plasma samples were prepared, aliquoted and stored at $-80^{\circ} \mathrm{C}$ until assayed. Total cholesterol, HDL-cholesterol and triglyceride levels were measured using a chromatographic enzymatic method in a Technicon automatic analyser RA-1000 (Dade Behring, Marburg, Germany). LDL-cholesterol was calculated using the Friedewald formula. Hypercholesterolemia was defined as total cholesterol levels $>200 \mathrm{mg} / \mathrm{dl}$ (or use of lipidlowering medications). Fasting blood glucose plasma levels were measured with a Beckman glucose analyzer (Beckman Instruments, Fullerton, CA, USA) and participants with levels $>125 \mathrm{mg} / \mathrm{dl}$ [or on antidiabetic medication(s)] were classified as having diabetes. For the purposes of this study, measurements were also performed for high sensitivity CRP plasma levels by particle-enhanced immunonephelometry [N Latex; Dade-Behring Marburg GmbH, Marburg, Germany; intra- and inter-assay coefficients of variation (CV): $<5 \%$ ], as well as for total adiponectin and high sensitivity IL-6 plasma levels by enzyme-linked immunosorbent assays (R\&D systems Inc, Minneapolis, Minnesota; intra- and inter-assay CV for both assays: $<10 \%$ ), according to the manufacturer's protocols. 
Finally, according to the results of the above baseline assessments, the study participants were also classified based on the presence or absence of metabolic syndrome at baseline according to the International Diabetes Federation (IDF) criteria/definition [20].

\section{ATTICA study 10-year follow-up (2002-2012) and cardiovascular disease incidence}

The ATTICA study 10-year follow-up was performed during 2011-2012 (median follow-up duration: 8.41 years), as previously described [21]. Data regarding the CVD health status of each study participant included development of: (a) myocardial infarction, angina pectoris, other identified forms of ischemia or coronary revascularization (i.e. coronary artery bypass surgery and percutaneous coronary intervention); (b) heart failure and chronic arrhythmias; and (c) stroke. Medical records and hospital data were obtained to confirm the CVD status of participants and the adjudication was performed by trained study physicians following the International Statistical Classification of Diseases and Related Health Problems 10th Revision (ICD10). For participants who died during the study follow-up period, the relevant information was acquired from death certificates and relatives. In the context of the present study, complete and clinically accurate data regarding the CVD status during the 10year study follow-up were obtained for 366 participants with measured baseline total adiponectin plasma levels (Table 1). Thus, herein we present the analyses of the adiponectin data from this working sub-sample in relation to the documented 10 -year incidence of fatal/non-fatal CVD.

\section{Statistical analysis}

Continuous variables are presented as mean values \pm standard deviation (SD), and categorical variables as frequencies. Normality assumption was assessed via P-P plots. Associations between categorical variables were tested using the chi-square test, while between continuous variables using Pearson $r$ or Spearman's rho coefficients for normally distributed and skewed variables, respectively. Comparisons of mean values of normally 
distributed variables between the study participants who developed CVD and the CVD-free participants were performed using Student's t-test, after controlling for equality of variances using the Levene's test. Comparisons of continuous variables without normal distribution were performed using the non-parametric Mann-Whitney U-test. Logistic regression models were applied to estimate the relative risk of CVD during the 10-year follow up in relation to baseline circulating adiponectin levels, including adjustments for multiple confounding factors, namely age, sex, BMI, waist circumference, smoking, physical activity level, Mediterranean diet adherence, hypertension, diabetes, hypercholesterolemia and key circulating pro-inflammatory biomarkers (i.e., IL-6 and CRP). The results of logistic regression models are presented as odds ratios (OR) and 95\% confidence intervals (CI). Survival analysis using Cox Proportional Hazards models was also applied, showing similar findings (data not shown in text), but the results from logistic regression were preferred to be presented because they showed more robust statistical estimators. All reported $p$ values are based on two-sided tests and the corresponding 95\% CI. The Statistical Package for Social Sciences (SPSS) version 22 (SPSS Inc, Chicago, IL, U.S.A.) was used for all statistical analyses.

\section{Results}

\section{Baseline characteristics and 10-year CVD incidence}

The key baseline characteristics of the study participants are summarized in Table 1. During the 10-year study follow-up, 27 of the 366 (7.4\%) participants with complete followup data and measured baseline adiponectin plasma levels had developed CVD; of these subjects $81 \%$ were men and 19\% women ( $p$ for gender difference: 0.01 ; Table 1 ). Compared to participants who remained CVD-free, the group of participants who developed CVD during the 10-year study follow-up consisted mainly of older men, and had more pack-years 
of smoking and higher baseline BMI and waist circumference values (all $p$ values $<0.05$; Table 1). This group further exhibited at baseline higher incidence of the various metabolic syndrome components (i.e., obesity, hypercholesterolemia, hypertension and diabetes) and higher incidence of the metabolic syndrome as defined by the IDF criteria, as well as worse fasting glycemic and lipidemic profiles and lower adherence to the Mediterranean diet (i.e. lower MedDietScore) (all $p$ values $<0.05$; Table 1 ).

In addition, participants who developed CVD during the study follow-up had significantly lower baseline total adiponectin plasma levels compared to those who remained CVD-free $(2.8 \pm 1.1$ vs. $4.0 \pm 1.9 \mu \mathrm{g} / \mathrm{ml}$, respectively; $p<0.001)$. Finally, these participants also had an enhanced pro-inflammatory profile at baseline, as indicated by the significantly higher circulating levels of both IL-6 $(p<0.001)$ and CRP $(p=0.02)$, compared to participants without CVD during the 10-year study follow-up (Table 1).

\section{Baseline associations between circulating adiponectin and key study variables}

Table 2 presents the correlations between the baseline total adiponectin plasma levels and selected key baseline variables in the study participants with complete 10-year follow-up and measured baseline total adiponectin plasma levels $(N=366)$ based on Spearman's rank correlation coefficient tests. Circulating adiponectin exhibited significant negative correlations with BMI, waist circumference, triglycerides, IL-6 and CRP levels (all $p$ values $<0.001$; Table 2), as well as with LDL-cholesterol and fasting glucose plasma levels

( $p=0.011$ and $p=0.002$ respectively; Table 2$)$. On the other hand, significant positive correlations were identified between circulating adiponectin and both the MedDietScore and HDL-cholesterol levels (both $p$ values $<0.001$; Table 2).

\section{Baseline circulating adiponectin levels and 10-year CVD incidence}

The results of the multi-adjusted logistic regression models applied to assess the likelihood (Odds ratio; 95\% CI) for the development of CVD during the 10-year study 
follow-up period in relation to the baseline circulating adiponectin levels are presented in Table 3. In the context of this study, after adjusting for age, sex, BMI and waist circumference, we found that an increase in circulating adiponectin levels by 1 unit was associated with reduced likelihood of CVD by $38 \%$ (OR: $0.62,95 \%$ CI: 0.42-0.91; Model 1; Table 3). This association remained unaffected by additional adjustments for other known CVD-risk related factors, including current smoking (pack-years of smoking were also included in the model to incorporate lifelong smoking habit, without any alterations in the results), physical activity (defined as reporting physical activities on the IPAQ) and Mediterranean diet adherence (Model 2), as well as for hypertension, diabetes and hypercholesterolemia (Model 3). Further adjusting for IL-6 plasma levels did not substantively affect this relationship, which was modestly attenuated by additional adjustment for circulating CRP (Model 4 and Model 5, respectively; Table 3).

\section{Discussion}

Our study presents long-term, prospective data showing that circulating adiponectin holds a significant inverse association with the 10-year CVD incidence in adults from the Greek general population without previous CVD, independently of several other established CVD risk factors. Indeed, in the context of the ATTICA study, this inverse association remained significant even after taking into account a range of factors related to CVD risk, including age, sex, BMI, waist circumference, smoking, physical activity, Mediterranean diet adherence, hypertension, diabetes and hypercholesterolemia. As such, this multi-adjusted logistic regression analysis showed that in our study sample an increment of 1 unit in circulating total adiponectin levels was associated with $36 \%$ lower CVD risk. Moreover, this association persisted and was essentially unaffected even after further adjustment for IL-6 plasma levels, while it was modestly attenuated by the additional adjustment for circulating 
CRP. This suggest that the mechanisms linking adiponectin to lower long-term CVD risk in our study population extend beyond the underlying low-grade inflammation, as expressed by these two key pro-inflammatory biomarkers.

Our study findings agree with previous epidemiological data indicating a protective association between circulating adiponectin and development of CVD [11, 22-31]. Following the initial study by Pischon et al. in 2004 which showed that higher plasma adiponectin levels were independently associated with lower risk of subsequent CHD in middle-aged CVD-free men, a number of subsequent studies have documented similar, at least to some extent, findings in various cohorts [22-29]. Furthermore, meta-analysis data from prospective studies with a follow-up period ranging from 4 to 20 years $(8$ nested case-control and 4 cohort studies; total of 14960 participants and 4132 incident CHD events) have also showed that higher circulating total adiponectin levels were independently associated with lower CHD risk in men and women [30]. Similarly, a CVD-protective effect of adiponectin was also noted in a meta-analysis of 17 case-control studies in Han Chinese subjects, showing that low total adiponectin circulating levels increased the risk of a first CVD event in the Han Chinese population [31].

This inverse association between circulating adiponectin and CVD can be attributed to a spectrum of adiponectin-induced anti-inflammatory, anti-atherogenic and cardiovascularprotective effects which can directly impact on endothelial function, atherosclerosis and cardiomyocytes [4-7, 32-34]. As such, anti-inflammatory adiponectin effects appear to play a key role against endothelial dysfunction and atherogenesis by inhibiting the pro-inflammatory cascade in endothelial cells [34-36]. Moreover, in these cells adiponectin can also suppress the excess production of reactive oxygen species induced by high-glucose conditions or oxidized-LDL, thus protecting the vasculature in T2DM [37]. Notably, adiponectin can further exert vasodilator actions by directly stimulating nitric oxide production in endothelial 
cells [38]. In addition, adiponectin may protect against the activation of the endothelial adhesion molecular cascade which facilitates the entry of macrophages into the vascular wall [39], and also suppress the macrophage to foam cell transformation process [40]. Finally, adiponectin has been shown to exert direct cardio-protective effects against ischemic myocardial injury, heart failure and cardiac hypertrophy [4, 32-34, 41-43]. Indeed, adiponectin can protect against myocardial ischemia/reperfusion injury through signaling pathways involving both anti-inflammatory and anti-apoptotic actions $[42,43]$. Interestingly, based on animal studies exploring cardiac remodeling after myocardial infarction, adiponectin may protect against systolic dysfunction following myocardial infarction by suppressing interstitial fibrosis and cardiac hypertrophy [44].

Despite the aforementioned prospective studies and the mounting experimental evidence supporting a direct protective role of circulating adiponectin in CVD, we must highlight that there is also a significant body of epidemiological data from studies that have found either a weaker than expected association between adiponectin plasma levels and CVD or no association at all $[12,45-55]$. Furthermore, there are also meta-analyses of prospective studies reporting lack of a direct association between circulating adiponectin and CHD or stroke $[12,55-57]$. This inconsistency and the conflicting findings in the literature can be attributed, at least in part, to differences between these studies in the design, studied population, follow-up duration and methodology (e.g. storing of samples and assays for adiponectin measurement), as well as to the various degrees of adjustment for confounding factors. For example, smoking is shown to influence the links between pro-inflammatory processes, metabolic syndrome and CVD [58], as well as circulating adiponectin levels [5961], thus adjustments for smoking habits are required when exploring these associations. However, it appears that additional factors implicated in the complex role of adiponectin in health and disease states are also responsible for the conflicting epidemiological data (e.g. 
circulating adiponectin forms, expression/role of adiponectin receptors, and regulation of adiponectin secretion/actions).

Of note, adiponectin undergoes post-translational modifications within adipocytes, resulting in multimerization $[62,63]$. As such, adiponectin is secreted as oligomers of trimers which circulate as low molecular weight (LMW: trimers or alternatively both trimers and hexamers), medium molecular weight (MMW: hexamers) and high molecular weight multimers (HMW: 18- to 36-mers) [3, 62, 63]. The latter predominates in plasma and appears to be more metabolically active $[62,63]$. Thus, in addition to differences regarding the assays used to measure adiponectin, measurement of different forms of circulating adiponectin (total or HMW) in various studies may also contribute to the noted inconsistency in reported findings.

Moreover, emerging evidence indicates that the adiponectin receptors further influence the circulating levels and role of adiponectin, particularly in relation to CVD [10, 32, 64, 65]. Adiponectin acts by binding to AdipoR1 (expressed ubiquitously; highest expression in skeletal muscle), AdipoR2 (expressed predominantly in the liver), and Tcadherin (a glycosylphosphatidylinositol-anchored extracellular protein without an intracellular domain, highly expressed in the heart, skeletal muscle and in endothelial and vascular smooth muscle cells) $[32,64,65]$. Both AdipoR1 and AdipoR2 are seventransmembrane receptors and are also expressed in endothelial and vascular smooth muscle cells, as well as in the heart where AdipoR1 has relatively greater expression than AdipoR2 $[32,64]$. Notably, T-cadherin is markedly expressed in endothelial and smooth muscle cells at sites of vascular injury and exhibits affinity only for the hexameric and HMW adiponectin forms [65]. As such, these adiponectin forms interact with T-cadherin and are abundantly present on vascular and muscle tissue surfaces where T-cadherin is highly expressed, forming a local reservoir $[32,64,66]$. This interaction is considered significant for the cardio- 
protective adiponectin effects and for the role of this adipokine in atherosclerosis $[10,32,64$, 66]. However, the exact mechanism(s) linking these receptors to the effects of adiponectin in the context of CVD remain to be clarified.

Notably, factors relating to the regulation of adiponectin synthesis/secretion, the adiponectin forms, and the adiponectin receptors may also be implicated in gender-specific differences noted in the relationship between circulating adiponectin and cardiometabolic complications $[11,22-24,46,49,67]$. Indeed, men appear to exhibit lower adiponectin plasma levels, potentially due to suppression by androgens [68]. However, women may also have lower circulating adiponectin levels after adjusting for body composition [69]. The latter indicates that the higher adiponectin plasma levels in women can be attributed to a more favorable body composition profile with less visceral/central adiposity [69]. As such, sex and body composition parameters should be taken into account when exploring the association of circulating adiponectin with CVD. Of note, opposing gender-specific associations between circulating HMW adiponectin and circulating T-cadherin levels have been noted in patients with stable coronary artery disease [70]. Moreover, different genotypes of adiponectin could be associated with higher CVD risk in a gender-specific way, since data suggest that the $\mathrm{C} / \mathrm{C}$ carriers of the rs266729 SNP of adiponectin may have increased CVD mortality risk compared to the $\mathrm{C} / \mathrm{G}$ or $\mathrm{G} / \mathrm{G}$ carriers in women, but not in men [71]. Overall, whether gender-specific associations of circulating adiponectin with cardiometabolic complications involve effects of sex hormones and/or sex-linked genes is not fully clarified yet and requires further research.

Finally, the so-called "adiponectin paradox" may also partly explain the conflicting evidence on the relationship between circulating adiponectin and CVD [10]. This paradox is noted mainly in older individuals and/or in certain patient groups (e.g. in patients with preexisting CHD, chronic heart failure or kidney disease) [10]. Indeed, studies in such 
populations have shown a positive association between high adiponectin plasma levels and increased mortality $[10,13-15]$. This may be attributed to different factors which are considered to play a role in the relationship of circulating adiponectin with CVD in healthy subjects and in certain disease states [10]. According to this hypothesis, in healthy young and middle-aged adults the inverse association between circulating adiponectin and CVD is driven by increased adiposity (particularly central adiposity) and its pathophysiologic consequences (e.g. obesity-related low-grade inflammation) [10]. On the other hand, factors relating to cachexia, involuntary weight loss, and sarcopenia are considered to contribute to increased secretion of adiponectin in older individuals and in certain chronic diseases [10]. These factors may partly account for the observed link between high adiponectin plasma levels and increased overall and CVD mortality in such populations [10]. In addition, other mechanisms which can also cause increased circulating adiponectin levels in these populations appear to include: ineffective compensatory up-regulation of adiponectin synthesis; decreased adiponectin signaling efficacy (biological resistance); decreased renal elimination of circulating adiponectin; altered expression of adiponectin receptors (e.g. of Tcadherin); and stimulated adiponectin secretion by certain CVD-related factors (e.g. by increased levels of natriuretic peptides) [10]. Moreover, adiponectin can be also produced by cardiomyocytes, myocytes and endothelial cells, and this non-adipose-derived adiponectin may also play a role in CVD $[3,10]$. It becomes evident that multiple factors, alone or in synergy, may significantly influence the association of circulating adiponectin with CVD and, hence, should be taken into account when interpreting the findings of studies in this field.

In this context, it is also noteworthy that "paradoxical hyperadiponectinemia" has also been found in certain obese individuals who are considered metabolically healthy (i.e., individuals with BMI over $30 \mathrm{~kg} / \mathrm{m}^{2}$ who are without obesity-related metabolic 
complications, such as T2DM, hypertension and dyslipidemia) [72-77]. These metabolically healthy obese subjects may exhibit paradoxically high adiponectin plasma levels despite the presence of obesity which is often long-standing and even severe/morbid [72-77]. Indeed, Aguilar-Salinas et al. have shown that $20 \%$ of obese adults (age range: $18-70$ years) with BMI over $40 \mathrm{~kg} / \mathrm{m}^{2}$ have total adiponectin plasma levels higher than the median level of subjects with normal BMI [77]. Moreover, this study showed that total adiponectin plasma levels which were paradoxically high over certain thresholds had a positive association with the metabolically healthy obese phenotype, even after controlling for age, waist circumference and fasting insulin levels [77]. Similar findings have been also reported in obese adolescent girls $[75,76]$. To what extent, in which specific patient groups (e.g. in which ethnic or age groups) and via which underlying mechanisms (e.g. links between adiponectin and the role of peripheral adiposity) this paradoxical hyperadiponectinemia may have a protective role against obesity-related cardiometabolic complications remains to be further elucidated [72-77].

\section{Strengths and limitations}

The present work has several strengths since the association between circulating adiponectin and CVD incidence was studied in the context of a prospective study with a long (10-year) follow-up period that focused specifically in adults from the general population without CVD history. Such epidemiological data are sparse from Mediterranean countries. Indeed, this is the first long-term, prospective study on CVD epidemiology in Greece, with a cohort which is representative of the urban Greek population (participants living in the greater Athens metropolitan area, i.e. an urban region, reflecting almost $70 \%$ of the total

Greek population). Furthermore, our study protocol involved assessments of multiple factors from all aspects of CVD risk determinants, including socio-demographic, lifestyle, clinical and biochemical variables. 
Despite these strengths, it is also important to acknowledge certain study limitations. As such, the relatively smaller sub-group of the ATTICA study participants with measured baseline circulating adiponectin levels could be considered a limitation of the present work. However, this sub-sample was representative of the total study's cohort without significant differences in the distributions of key factors. The low incidence of CVD noted in this study could be another limitation of the present analysis, but this was mainly due to the ATTICA study sampling scheme (i.e. enrolling participants from all age groups, representative to the total Greek population and without prior CVD); but this could be also viewed as a relative advantage, since it may reflect more precisely the true effect size of the studied factors on development of CVD in the general population compared to other studies that enrolled mainly participants of older age and/or participants with pre-existing CVD. Finally, based on our study protocol, we measured total levels of circulating adiponectin without selectively measuring HMW adiponectin plasma levels.

\section{Conclusion}

Our study shows that elevated circulating total adiponectin levels are independently associated with lower 10 -year CVD risk in adults without previous CVD. This association appears to be modestly attenuated by circulating CRP levels, but it was not mediated by IL-6 which is the main endocrine/circulating pro-inflammatory cytokine. Our present findings add to the body of existing evidence on the relationship between total adiponectin plasma levels and incident CVD, and offer new data for a population that has been under-represented in long-term cardiovascular epidemiology studies. 


\section{Acknowledgements}

The authors would like to thank the ATTICA study group of investigators: Yannis Skoumas, Natasa Katinioti, Labros Papadimitriou, Constantina Masoura, Spiros Vellas, Yannis Lentzas, Manolis Kambaxis, Konstanitna Paliou, Vassiliki Metaxa, Agathi Ntzouvani, Dimitris Mpougatas, Nikolaos Skourlis, Christina Papanikolaou, Aikaterini Kalogeropoulou, Evangelia Pitaraki, Alexandros Laskaris, Mihail Hatzigeorgiou, Athanasios Grekas, and Eleni Kokkou for assistance in the initial physical examination and/or the follow-up evaluation; Efi Tsetsekou for her assistance in psychological evaluation and follow-up evaluation, as well as the laboratory team: Carmen Vassiliadou and George Dedousis (genetic analysis), Marina Toutouza-Giotsa, Constantina Tselika and Sia Poulopoulou (biochemical analysis), and Maria Toutouza for the database management.

Funding: The ATTICA study is supported by research grants from the Hellenic Cardiology Society (HCS2002) and the Hellenic Atherosclerosis Society (HAS2003). Demosthenes Panagiotakos and Ekavi Georgousopoulou have received grants by the Coca-Cola Company.

Conflict of interest: The authors declare that they have no conflict of interest.

Ethical approval: All procedures performed in studies involving human participants were in accordance with the ethical standards of the institutional and/or national research committee and with the 1964 Helsinki declaration and its later amendments or comparable ethical standards. 


\section{References}

1) Galic S, Oakhill JS, Steinberg GR. Adipose tissue as an endocrine organ. Mol Cell Endocrinol. 25;316(2):129-39 (2010)

2) Rondinone CM. Adipocyte-derived hormones, cytokines, and mediators. Endocrine. 29(1):81-90 (2006)

3) Fasshauer M, Blüher M. Adipokines in health and disease. Trends Pharmacol Sci. 36(7):461-70 (2015)

4) Kyrou I, Mattu HS, Chatha K, Randeva HS. Chapter 7 - Fat Hormones, Adipokines. In Schisler JC, Lang CH, Willis MS (eds) Endocrinology of the Heart in health and disease. pp 167-205. Academic Press (2017)

5) Brochu-Gaudreau K, Rehfeldt C, Blouin R, Bordignon V, Murphy BD, Palin MF. Adiponectin action from head to toe. Endocrine. 37(1):11-32 (2010)

6) Ahima RS. Metabolic actions of adipocyte hormones: focus on adiponectin. Obesity (Silver Spring). 14 Suppl 1:9S-15S (2006)

7) Turer AT, Scherer PE. Adiponectin: mechanistic insights and clinical implications. Diabetologia. 55(9):2319-26 (2012)

8) Yousuf O, Mohanty BD, Martin SS, Joshi PH, Blaha MJ, Nasir K, Blumenthal RS, Budoff MJ. High-sensitivity C-reactive protein and cardiovascular disease: a resolute belief or an elusive link? J Am Coll Cardiol. 30;62(5):397-408 (2013)

9) Yudkin JS, Kumari M, Humphries SE, Mohamed-Ali V. Inflammation, obesity, stress and coronary heart disease: is interleukin-6 the link? Atherosclerosis. 148(2):209-14 (2000)

10) Kizer JR. Adiponectin, cardiovascular disease, and mortality: parsing the dual prognostic implications of a complex adipokine. Metabolism. 63(9):1079-83 (2014)

11) Pischon T, Girman CJ, Hotamisligil GS, Rifai N, Hu FB, Rimm EB. Plasma adiponectin levels and risk of myocardial infarction in men. JAMA. 14;291(14):1730-7 (2004)

12) Sattar N, Wannamethee G, Sarwar N, Tchernova J, Cherry L, Wallace AM, Danesh J, Whincup PH. Adiponectin and coronary heart disease: a prospective study and meta-analysis. Circulation. 15;114(7):623-9 (2006)

13) Poehls J, Wassel CL, Harris TB, Havel PJ, Swarbrick MM, Cummings SR, Newman AB, Satterfield S, Kanaya AM; Health ABC Study. Association of adiponectin with mortality in older adults: the Health, Aging, and Body Composition Study. Diabetologia. 52(4):591-5 (2009)

14) Cavusoglu E, Ruwende C, Chopra V, Yanamadala S, Eng C, Clark LT, Pinsky DJ, Marmur JD. Adiponectin is an independent predictor of all-cause mortality, cardiac mortality, 
and myocardial infarction in patients presenting with chest pain. Eur Heart J. 27(19):2300-9 (2006)

15) Kistorp C, Faber J, Galatius S, Gustafsson F, Frystyk J, Flyvbjerg A, Hildebrandt P. Plasma adiponectin, body mass index, and mortality in patients with chronic heart failure. Circulation. 20;112(12):1756-62 (2005)

16) Pitsavos C, Panagiotakos DB, Chrysohoou C, Stefanadis C. Epidemiology of cardiovascular risk factors in Greece: aims, design and baseline characteristics of the ATTICA study. BMC Public Health. 20;3:32 (2003)

17) Katsouyanni K, Rimm EB, Gnardellis C, Trichopoulos D, Polychronopoulos E, Trichopoulou A. Reproducibility and relative validity of an extensive semi-quantitative food frequency questionnaire using dietary records and biochemical markers among Greek schoolteachers. Int J Epidemiol. 26 Suppl 1:S118-27 (1997)

18) Panagiotakos DB, Pitsavos C, Stefanadis C. Dietary patterns: a Mediterranean diet score and its relation to clinical and biological markers of cardiovascular disease risk. Nutr Metab Cardiovasc Dis. 16(8):559-68 (2006)

19) Papathanasiou G, Georgoudis G, Papandreou M, Spyropoulos P, Georgakopoulos D, Kalfakakou V, Evangelou A. Reliability measures of the short International Physical Activity Questionnaire (IPAQ) in Greek young adults. Hellenic J Cardiol. 50(4):283-94 (2009)

20) Alberti KG, Zimmet P, Shaw J; IDF Epidemiology Task Force Consensus Group. The metabolic syndrome--a new worldwide definition. Lancet. Sep 24-30;366(9491):1059-62 (2005)

21) Panagiotakos DB, Georgousopoulou EN, Pitsavos C, Chrysohoou C, Metaxa V, Georgiopoulos GA, Kalogeropoulou K, Tousoulis D, Stefanadis C; ATTICA Study group. Ten-year (2002-2012) cardiovascular disease incidence and all-cause mortality, in urban Greek population: the ATTICA Study. Int J Cardiol. 1;180:178-84 (2015)

22) Rothenbacher D, Brenner H, März W, Koenig W. Adiponectin, risk of coronary heart disease and correlations with cardiovascular risk markers. Eur Heart J. 26 (16):1640-6 (2005) 23) Pischon T, Hu FB, Girman CJ, Rifai N, Manson JE, Rexrode KM, Rimm EB. Plasma total and high molecular weight adiponectin levels and risk of coronary heart disease in women. Atherosclerosis. 219(1):322-9 (2011)

24) Frystyk J, Berne C, Berglund L, Jensevik K, Flyvbjerg A, Zethelius B. Serum adiponectin is a predictor of coronary heart disease: a population-based 10-year follow-up study in elderly men. J Clin Endocrinol Metab. 92(2):571-6 (2007) 
25) Ho DY, Cook NR, Britton KA, Kim E, Creager MA, Ridker PM, Pradhan AD. Highmolecular-weight and total adiponectin levels and incident symptomatic peripheral artery disease in women: a prospective investigation. Circulation. 22;124(21):2303-11 (2011)

26) Saito I, Yamagishi K, Chei CL, Cui R, Ohira T, Kitamura A, Kiyama M, Imano H, Okada T, Kato T, Hitsumoto S, Ishikawa Y, Tanigawa T, Iso H. Total and high molecular weight adiponectin levels and risk of cardiovascular disease in individuals with high blood glucose levels. Atherosclerosis. 229(1):222-7 (2013)

27) Schulze MB, Shai I, Rimm EB, Li T, Rifai N, Hu FB. Adiponectin and future coronary heart disease events among men with type 2 diabetes. Diabetes. 54(2):534-9 (2005)

28) Kumada M, Kihara S, Sumitsuji S, Kawamoto T, Matsumoto S, Ouchi N, Arita Y, Okamoto Y, Shimomura I, Hiraoka H, Nakamura T, Funahashi T, Matsuzawa Y; Osaka CAD Study Group. Coronary artery disease. Association of hypoadiponectinemia with coronary artery disease in men. Arterioscler Thromb Vasc Biol. 1;23(1):85-9 (2003)

29) Ai M, Otokozawa S, Asztalos BF, White CC, Cupples LA, Nakajima K, Lamon-Fava S, Wilson PW, Matsuzawa Y, Schaefer EJ. Adiponectin: an independent risk factor for coronary heart disease in men in the Framingham offspring Study. Atherosclerosis. 217(2):543-8 (2011)

30) Zhang H, Mo X, Hao Y, Huang J, Lu X, Cao J, Gu D. Adiponectin levels and risk of coronary heart disease: a meta-analysis of prospective studies. Am J Med Sci. 345(6):455-61 (2013)

31) Zhang BC, Liu WJ, Che WL, Xu YW. Serum total adiponectin level and risk of cardiovascular disease in Han Chinese populations: a meta-analysis of 17 case-control studies. Clin Endocrinol (Oxf). 77(3):370-8 (2012)

32) Parker-Duffen JL, Walsh K. Cardiometabolic effects of adiponectin. Best Pract Res Clin Endocrinol Metab. 28(1):81-91 (2014)

33) Caselli C, D'Amico A, Cabiati M, Prescimone T, Del Ry S, Giannessi D. Back to the heart: the protective role of adiponectin. Pharmacol Res. 82:9-20 (2014)

34) Hopkins TA, Ouchi N, Shibata R, Walsh K. Adiponectin actions in the cardiovascular system. Cardiovasc Res. 1;74(1):11-8 (2007)

35) Ouchi N, Kihara S, Arita Y, Okamoto Y, Maeda K, Kuriyama H, Hotta K, Nishida M, Takahashi M, Muraguchi M, Ohmoto Y, Nakamura T, Yamashita S, Funahashi T, Matsuzawa Y. Adiponectin, an adipocyte-derived plasma protein, inhibits endothelial NFkappaB signaling through a cAMP-dependent pathway. Circulation. 12;102(11):1296-301 (2000) 
36) Hattori Y, Nakano Y, Hattori S, Tomizawa A, Inukai K, Kasai K. High molecular weight adiponectin activates AMPK and suppresses cytokine-induced NF-kappaB activation in vascular endothelial cells. FEBS Lett. 28;582(12):1719-24 (2008)

37) Ouedraogo R, Wu X, Xu SQ, Fuchsel L, Motoshima H, Mahadev K, Hough K, Scalia R, Goldstein BJ. Adiponectin suppression of high-glucose-induced reactive oxygen species in vascular endothelial cells: evidence for involvement of a cAMP signaling pathway. Diabetes. Jun;55(6):1840-6 (2006)

38) Chen H, Montagnani M, Funahashi T, Shimomura I, Quon MJ. Adiponectin stimulates production of nitric oxide in vascular endothelial cells. J Biol Chem. 7;278(45):45021-6 (2003)

39) Ouedraogo R, Gong Y, Berzins B, Wu X, Mahadev K, Hough K, Chan L, Goldstein BJ, Scalia R. Adiponectin deficiency increases leukocyte-endothelium interactions via upregulation of endothelial cell adhesion molecules in vivo. J Clin Invest. 117(6):1718-26 (2007)

40) Ouchi N, Kihara S, Arita Y, Nishida M, Matsuyama A, Okamoto Y, Ishigami M, Kuriyama H, Kishida K, Nishizawa H, Hotta K, Muraguchi M, Ohmoto Y, Yamashita S, Funahashi T, Matsuzawa Y. Adipocyte-derived plasma protein, adiponectin, suppresses lipid accumulation and class A scavenger receptor expression in human monocyte-derived macrophages. Circulation. Feb 27;103(8):1057-63 (2001)

41) Karmazyn M, Purdham DM, Rajapurohitam V, Zeidan A. Signalling mechanisms underlying the metabolic and other effects of adipokines on the heart. Cardiovasc Res. 15;79(2):279-86 (2008)

42) Ouchi N, Shibata R, Walsh K. Cardioprotection by adiponectin. Trends Cardiovasc Med. 16(5):141-6 (2006)

43) Shibata R, Sato K, Pimentel DR, Takemura Y, Kihara S, Ohashi K, Funahashi T, Ouchi N, Walsh K. Adiponectin protects against myocardial ischemia-reperfusion injury through AMPK- and COX-2-dependent mechanisms. Nat Med. 11(10):1096-103 (2005)

44) Shibata R, Izumiya Y, Sato K, Papanicolaou K, Kihara S, Colucci WS, Sam F, Ouchi N, Walsh K. Adiponectin protects against the development of systolic dysfunction following myocardial infarction. J Mol Cell Cardiol. 42(6):1065-74 (2007)

45) Lindsay RS, Resnick HE, Zhu J, Tun ML, Howard BV, Zhang Y, Yeh J, Best LG. Adiponectin and coronary heart disease: the Strong Heart Study. Arterioscler Thromb Vasc Biol. 25(3):e15-6 (2005) 
46) Laughlin GA, Barrett-Connor E, May S, Langenberg C. Association of adiponectin with coronary heart disease and mortality: the Rancho Bernardo study. Am J Epidemiol. $15 ; 165(2): 164-74(2007)$

47) Côté M, Cartier A, Reuwer AQ, Arsenault BJ, Lemieux I, Després JP, Wareham NJ, Kastelein JJ, Boekholdt SM, Khaw KT. Adiponectin and risk of coronary heart disease in apparently healthy men and women (from the EPIC-Norfolk Prospective Population Study). Am J Cardiol. Aug 1;108(3):367-73 (2011)

48) Koenig W, Khuseyinova N, Baumert J, Meisinger C, Löwel H. Serum concentrations of adiponectin and risk of type 2 diabetes mellitus and coronary heart disease in apparently healthy middle-aged men: results from the 18-year follow-up of a large cohort from southern Germany. J Am Coll Cardiol. 3;48(7):1369-77 (2006)

49) Lawlor DA, Davey Smith G, Ebrahim S, Thompson C, Sattar N. Plasma adiponectin levels are associated with insulin resistance, but do not predict future risk of coronary heart disease in women. J Clin Endocrinol Metab. 90(10):5677-83 (2005)

50) Kuller LH, Grandits G, Cohen JD, Neaton JD, Prineas R; Multiple Risk Factor Intervention Trial Research Group. Lipoprotein particles, insulin, adiponectin, C-reactive protein and risk of coronary heart disease among men with metabolic syndrome. Atherosclerosis. 195(1):122-8 (2007)

51) Matsumoto M, Ishikawa S, Kajii E. Association of adiponectin with cerebrovascular disease: a nested case-control study. Stroke. 39(2):323-8 (2008)

52) Sattar N, Watt P, Cherry L, Ebrahim S, Davey Smith G, Lawlor DA. High molecular weight adiponectin is not associated with incident coronary heart disease in older women: a nested prospective case-control study. J Clin Endocrinol Metab. 93(5):1846-9 (2008)

53) Hatano Y, Matsumoto M, Ishikawa S, Kajii E. Plasma adiponectin level and myocardial infarction: the JMS Cohort Study. J Epidemiol. 19(2):49-55 (2009)

54) Ogorodnikova AD, Wassertheil-Smoller S, Mancuso P, Sowers MR, Rajpathak SN, Allison MA, Baird AE, Rodriguez B, Wildman RP. High-molecular-weight adiponectin and incident ischemic stroke in postmenopausal women: a Women's Health Initiative Study. Stroke. 41(7):1376-81 (2010)

55) Arregui M, Buijsse B, Fritsche A, di Giuseppe R, Schulze MB, Westphal S, Isermann B, Boeing H, Weikert C. Adiponectin and risk of stroke: prospective study and meta-analysis. Stroke. 45(1):10-7 (2014) 
56) Sook Lee E, Park SS, Kim E, Sook Yoon Y, Ahn HY, Park CY, Ho Yun Y, Woo Oh S. Association between adiponectin levels and coronary heart disease and mortality: a systematic review and meta-analysis. Int J Epidemiol. 42(4):1029-39 (2013)

57) Kanhai DA, Kranendonk ME, Uiterwaal CS, van der Graaf Y, Kappelle LJ, Visseren FL. Adiponectin and incident coronary heart disease and stroke. A systematic review and metaanalysis of prospective studies. Obes Rev. 14(7):555-67 (2013)

58) Cena H, Fonte ML, Turconi G. Relationship between smoking and metabolic syndrome. Nutr Rev. Dec;69(12):745-53 (2011)

59) Tsai JS, Guo FR, Chen SC, Lue BH, Chiu TY, Chen CY, Hung SH, Chuang LM, Chen CY. Smokers show reduced circulating adiponectin levels and adiponectin mRNA expression in peripheral blood mononuclear cells. Atherosclerosis. Sep;218(1):168-73 (2011)

60) Kotani K, Hazama A, Hagimoto A, Saika K, Shigeta M, Katanoda K, Nakamura M. Adiponectin and smoking status: a systematic review. J Atheroscler Thromb. 19(9):787-94 (2012)

61) Kryfti M, Dimakou K, Toumbis M, Daniil Z, Hatzoglou C, Gourgoulianis KI. Effects of smoking cessation on serum leptin and adiponectin levels. Tob Induc Dis. Sep 3;13:30 (2015) 62) Simpson F, Whitehead JP. Adiponectin--it's all about the modifications. Int J Biochem Cell Biol. 42(6):785-8 (2010)

63) Wang Y, Lam KS, Yau MH, Xu A. Post-translational modifications of adiponectin: mechanisms and functional implications. Biochem J. 1;409(3):623-33 (2008)

64) Yamauchi T, Iwabu M, Okada-Iwabu M, Kadowaki T. Adiponectin receptors: a review of their structure, function and how they work. Best Pract Res Clin Endocrinol Metab. 28(1):15-23 (2014)

65) Hug C, Wang J, Ahmad NS, Bogan JS, Tsao TS, Lodish HF. T-cadherin is a receptor for hexameric and high-molecular-weight forms of Acrp30/adiponectin. Proc Natl Acad Sci U S A. $13 ; 101(28): 10308-13$ (2004)

66) Denzel MS, Scimia MC, Zumstein PM, Walsh K, Ruiz-Lozano P, Ranscht B. T-cadherin is critical for adiponectin-mediated cardioprotection in mice. $\mathrm{J}$ Clin Invest. Dec;120(12):4342-52 (2010)

67) Menzaghi C, Xu M, Salvemini L, De Bonis C, Palladino G, Huang T, Copetti M, Zheng Y, Li Y, Fini G, Hu FB, Bacci S, Qi L, Trischitta V. Circulating adiponectin and cardiovascular mortality in patients with type 2 diabetes mellitus: evidence of sexual dimorphism. Cardiovasc Diabetol. Sep 10;13:130 (2014) 
68) Nishizawa H, Shimomura I, Kishida K, Maeda N, Kuriyama H, Nagaretani H, Matsuda M, Kondo H, Furuyama N, Kihara S, Nakamura T, Tochino Y, Funahashi T, Matsuzawa Y. Androgens decrease plasma adiponectin, an insulin-sensitizing adipocyte-derived protein. Diabetes. Sep;51(9):2734-41 (2002)

69) Lew J, Sanghavi M, Ayers CR, McGuire DK, Omland T, Atzler D, Gore MO, Neeland I, Berry JD, Khera A, Rohatgi A, de Lemos JA. Sex-Based Differences in Cardiometabolic Biomarkers. Circulation. Feb 7;135(6):544-555 (2017)

70) Schoenenberger AW, Pfaff D, Dasen B, Frismantiene A, Erne P, Resink TJ, Philippova M. Gender-Specific Associations between Circulating T-Cadherin and High Molecular Weight-Adiponectin in Patients with Stable Coronary Artery Disease. PLoS One. Jun 17;10(6):e0131140 (2015)

71) Alehagen U, Vorkapic E, Ljungberg L, Länne T, Wågsäter D. Gender difference in adiponectin associated with cardiovascular mortality. BMC Med Genet. Jun 12;16:37 (2015) 72) Muñoz-Garach A, Cornejo-Pareja I, Tinahones FJ. Does Metabolically Healthy Obesity Exist? Nutrients. Jun 1;8(6) (2016)

73) Ahl S, Guenther M, Zhao S, James R, Marks J, Szabo A, Kidambi S. Adiponectin Levels Differentiate Metabolically Healthy vs Unhealthy Among Obese and Nonobese White Individuals. J Clin Endocrinol Metab. Nov;100(11):4172-80 (2015)

74) Doumatey AP, Bentley AR, Zhou J, Huang H, Adeyemo A, Rotimi CN. Paradoxical Hyperadiponectinemia is Associated With the Metabolically Healthy Obese (MHO) Phenotype in African Americans. J Endocrinol Metab. Apr 1;2(2):51-65 (2012)

75) Morrison JA, Glueck CJ, Daniels S, Wang P, Stroop D. Paradoxically high adiponectin in obese 16-year-old girls protects against appearance of the metabolic syndrome and its components seven years later. J Pediatr. Feb;158(2):208-14.e1 (2011)

76) Morrison JA, Glueck CJ, Daniels S, Wang P, Horn P, Stroop D. Paradoxically high adiponectin and the healthy obese phenotype in obese black and white 16-year-old girls. Transl Res. Nov;156(5):302-8 (2010)

77) Aguilar-Salinas CA, García EG, Robles L, Riaño D, Ruiz-Gomez DG, García-Ulloa AC, Melgarejo MA, Zamora M, Guillen-Pineda LE, Mehta R, Canizales-Quinteros S, Tusie Luna MT, Gómez-Pérez FJ. High adiponectin concentrations are associated with the metabolically healthy obese phenotype. J Clin Endocrinol Metab. Oct;93(10):4075-9 (2008) 
Table 1. Selected key baseline characteristics for the total study sample $(N=531)$ and for all participants with complete 10-year follow-up and measured baseline total adiponectin plasma levels $(N=366)$ based on the development of fatal or non-fatal cardiovascular disease (CVD) during the study follow-up period.

\begin{tabular}{|c|c|c|c|c|c|}
\hline \multirow[b]{2}{*}{ Baseline characteristics } & \multirow{2}{*}{$\begin{array}{c}\text { Total study } \\
\text { sample } \\
(N=\mathbf{5 3 1})\end{array}$} & \multicolumn{3}{|c|}{$\begin{array}{l}\text { Study participants with confirmed CVD status at } \\
\text { the } 10 \text {-year follow-up }\end{array}$} & \multirow[b]{2}{*}{$\boldsymbol{P}$} \\
\hline & & $\begin{array}{l}\text { All follow-up } \\
\qquad(N=366)\end{array}$ & $\begin{array}{l}\text { CVD-free } \\
(N=339)\end{array}$ & $\begin{array}{l}\text { Fatal/non-fatal CVD } \\
\qquad(N=27)\end{array}$ & \\
\hline Age (years) & $40 \pm 11$ & $40 \pm 12$ & $39 \pm 11$ & $55 \pm 9$ & $<0.001$ \\
\hline Gender, \% male & 58 & 58 & 56 & 81 & 0.01 \\
\hline Education (years) & $12.4 \pm 3.6$ & $12.4 \pm 3.6$ & $12.5 \pm 3.5$ & $11.3 \pm 4.3$ & 0.23 \\
\hline BMI $\left(\mathrm{kg} / \mathrm{m}^{2}\right)$ & $25.9 \pm 4.4$ & $26.1 \pm 4.5$ & $25.9 \pm 4.5$ & $29.1 \pm 4.1$ & $<0.001$ \\
\hline Waist circumference (cm) & $89 \pm 16$ & $90 \pm 16$ & $89 \pm 15$ & $102 \pm 13$ & $<0.001$ \\
\hline Current smokers (\% Yes) & 40 & 41 & 41 & 35 & 0.03 \\
\hline Ex-smokers (\%Yes) & 18 & 17 & 15 & 43 & 0.02 \\
\hline Years stopped smoking & $9.8 \pm 8.1$ & $10.1 \pm 9.9$ & $9.5 \pm 8.2$ & $12.2 \pm 11.2$ & 0.077 \\
\hline Pack-years of smoking & $496 \pm 501$ & $501 \pm 500$ & $441 \pm 426$ & $760 \pm 704$ & $<0.001$ \\
\hline Physical activity status* (\% Yes) & 38 & 40 & 40 & 33 & 0.47 \\
\hline Minimally active (\% Yes) & 25 & 26 & 26 & 23 & \\
\hline HEPA active (\% Yes) & 13 & 14 & 14 & 10 & \\
\hline MedDietScore (range: $0-55$ ) & $27 \pm 6$ & $27 \pm 5$ & $27 \pm 6$ & $23 \pm 5$ & $<0.001$ \\
\hline Obesity (\% Yes) & 17 & 18 & 17 & 33 & 0.04 \\
\hline Hypertension (\% Yes) & 26 & 29 & 26 & 59 & $<0.001$ \\
\hline Diabetes (\% Yes) & 3 & 3 & 2 & 19 & $<0.001$ \\
\hline Hypercholesterolemia (\% Yes) & 37 & 40 & 37 & 78 & $<0.001$ \\
\hline Metabolic Syndrome** (\% Yes) & 45 & 47 & 45 & 70 & 0.01 \\
\hline Fasting Glucose (mg/dl) & $89 \pm 20$ & $90 \pm 19$ & $89 \pm 17$ & $102 \pm 31$ & 0.01 \\
\hline Total Cholesterol (mg/dl) & $189 \pm 37$ & $191 \pm 38$ & $188 \pm 37$ & $220 \pm 41$ & $<0.001$ \\
\hline Triglycerides (mg/dl) & $107 \pm 72$ & $108 \pm 67$ & $104 \pm 64$ & $165 \pm 78$ & $<0.001$ \\
\hline HDL cholesterol (mg/dl) & $48 \pm 14$ & $48 \pm 13$ & $49 \pm 13$ & $40 \pm 13$ & $<0.001$ \\
\hline LDL cholesterol (mg/dl) & $120 \pm 35$ & $121 \pm 35$ & $118 \pm 34$ & $147 \pm 39$ & $<0.001$ \\
\hline Adiponectin $(\mu \mathrm{g} / \mathrm{ml})$ & $4.0 \pm 2.0$ & $3.9 \pm 1.9$ & $4.0 \pm 1.9$ & $2.8 \pm 1.1$ & $<0.001$ \\
\hline IL-6 (pg/ml) & $1.4 \pm 0.3$ & $1.4 \pm 0.3$ & $1.4 \pm 0.4$ & $1.6 \pm 0.2$ & $<0.001$ \\
\hline CRP (mg/L) & $1.8 \pm 2.1$ & $1.8 \pm 2.2$ & $1.7 \pm 2.1$ & $2.5 \pm 2.1$ & 0.02 \\
\hline
\end{tabular}

Data presented as means \pm standard deviation for continuous variables, and as frequencies for categorical variables. $P$ values for comparisons between CVD-free participants and those with confirmed CVD at the 10-year follow-up derived using the Mann-Whitney test for continuous variables and the chi-square test for categorical variables.

*: Physical activity status: physically active participants categorized as minimally active and healthy enhancing physical active (HEPA) based on the International Physical Activity Questionnaire (IPAQ) [19].

**: Metabolic syndrome defined based on the International Diabetes Federation (IDF) criteria/definition [20].

CVD: cardiovascular disease; BMI: body mass index; WC: waist circumference; MedDietScore: score evaluating the adherence to the Mediterranean diet (higher score values indicate better adherence); IL-6: interleukin 6; CRP: Creactive protein 
Table 2. Correlations between circulating total adiponectin levels and selected baseline variables in the participants with complete 10-year follow-up and measured baseline adiponectin plasma levels $(N=366)$ tested by the Spearman's rank correlation coefficient.

\begin{tabular}{|c|c|c|}
\hline \multirow[t]{2}{*}{ Baseline characteristics } & \multicolumn{2}{|c|}{ Circulating total adiponectin levels } \\
\hline & $r$ & $P$ value \\
\hline BMI $\left(\mathbf{k g} / \mathbf{m}^{2}\right)$ & -0.336 & $<0.001$ \\
\hline Waist circumference $(\mathrm{cm})$ & -0.389 & $<0.001$ \\
\hline Fasting Glucose (mg/dl) & -0.161 & 0.002 \\
\hline Total cholesterol (mg/dl) & -0.088 & 0.096 \\
\hline Triglycerides (mg/dl) & -0.319 & $<0.001$ \\
\hline LDL cholesterol (mg/dl) & -0.136 & 0.011 \\
\hline HDL cholesterol (mg/dl) & 0.431 & $<0.001$ \\
\hline MedDietScore (range: 0-55) & 0.343 & $<0.001$ \\
\hline IL-6 (pg/ml) & -0.181 & 0.001 \\
\hline CRP (mg/L) & -0.175 & 0.001 \\
\hline Pack-years of smoking & -0.067 & 0.250 \\
\hline \multicolumn{3}{|c|}{$\begin{array}{l}\text { BMI: body mass index; WC: waist circumference; MedDietScore: score } \\
\text { evaluating the adherence to the Mediterranean diet (higher score values } \\
\text { indicate better adherence); IL-6: interleukin 6; CRP: C-reactive protein }\end{array}$} \\
\hline
\end{tabular}


Table 3. Results from logistic regression analysis models estimating the relative risk (Odds ratio; 95\% Confidence Intervals) for the development of a cardiovascular disease event (outcome) during the 10-year study follow-up period according to the baseline circulating total adiponectin levels.

\begin{tabular}{|c|c|c|}
\hline \multirow{2}{*}{ Model } & $\begin{array}{r}\text { 10-year risk of a CVD event (outcome) according } \\
\text { to the baseline circulating adiponectin levels }\end{array}$ & P value \\
\hline $\mathbf{1}$ & Odds ratio (95\% CI) & 0.01 \\
\hline $\mathbf{2}$ & $0.62(0.42-0.91)$ & 0.02 \\
\hline $\mathbf{3}$ & $0.63(0.43-0.92)$ & 0.03 \\
\hline $\mathbf{4}$ & $0.64(0.42-0.96)$ & 0.03 \\
\hline $\mathbf{5}$ & $0.6(0.38-0.94)$ & 0.046 \\
\hline
\end{tabular}

Model 1: adjusted for age, sex, body mass index, and waist circumference

Model 2: Model 1 additionally adjusted for current smoking (pack-years of smoking were also included to incorporate lifelong smoking habit, without any significant change in the results), physical activity and MedDietScore

Model 3: Model 2 additionally adjusted for hypertension, diabetes, and hypercholesterolemia Model 4: Model 3 additionally adjusted for circulating Interleukin-6 levels Model 5: Model 3 additionally adjusted for circulating C-reactive protein levels CI: Confidence Intervals; CVD: cardiovascular disease; MedDietScore: score evaluating the adherence to the Mediterranean diet (higher score values indicate better adherence) 